\title{
Drag performance of divergent tubular-truncated cones: a shape optimization study
}

\author{
A. Lotfi $\cdot$ M. Rad
}

Received: 7 March 2011/Revised: 3 April 2011/Accepted: 8 September 2011/Published online: 23 November 2011

(c) CEERS, IAU 2011

\begin{abstract}
The use of more efficient energy consuming devices, which are closely associated with reduction of environmental pollution, has gained significant interest in the recent decades. The reduction of drag coefficient also improves safety and durability of environmental structures subjected to high-velocity fluid flow, and causes the noise and vibration to decrease as well. This paper describes the efficiency improvement in energy management by means of reducing drag coefficient in a practical divergent tubular-truncated cone. Extensive numerical simulations with emphasis on the shape optimization study were performed in order to find minimum drag coefficient for both laminar and turbulent flows $\left(9.41 \times 10^{2} \leq \mathrm{Re} \leq 1.882 \times 10^{7}\right)$ around the mentioned cone. The numerical results were validated with experimental data, obtained by performing tests in an open circuit wind tunnel. The results showed that the minimum drag coefficient of optimum model within the shape modification process, comparable to the value for the primary model in turbulent flow decreased by $69.8 \%$ (the maximum discrepancy) at the highest considered Reynolds number of $1.882 \times 10^{7}$. Furthermore, the study of streamwise velocity profile led to more useful result. In this regard, the velocity magnitude inside the inlet span of the primary model, close to the entrance was approximately 1.6 times as great as the free upstream
\end{abstract}

\footnotetext{
A. Lotfi $(\bowtie)$

Department of Mechanical Engineering, Islamic Azad University, South Tehran Branch,

No. 209, North Iranshahr St., Tehran, Iran

e-mail: lotfi.azadeh@gmail.com

M. Rad

Department of Mechanical Engineering,

Sharif University of Technology, Azadi Ave., Tehran, Iran
}

velocity. This is a noticeable result for the use of efficiency important structures in industrial applications.

Keywords Computational fluid dynamics · Drag reduction - Optimum shape $\cdot$ Velocity profile $\cdot$ Wind tunnel

\section{Introduction}

The drag is a force a flowing fluid exerts on a body in the flow direction. It is usually recognized as an undesirable effect, like friction, and the first thought that comes to mind is to minimize it. It is also strongly dependent on the shape of the body and any effect that causes the shape to change has a profound effect on it (Cengel and Cimbala 2010). It may also reduce the efficiency considerably in non-optimized designed bodies. Moreover, the decrease of drag coefficient over various bodies is strongly associated with the reduction of fuel and energy consumption and thus considerable decrease in their costs, as one of the main concerns in the recent decades. Furthermore, the saving on fuel for use of the energy management approaches caused by drag reduction has resulted in using smaller and more efficient propulsion systems. On one hand, the manufacturing and installation costs become much lower by utilizing more efficient propulsion systems. On the other hand, less fuel consumption leads to the noticeable decrease in environmental pollution that is really of consequence. Reducing drag also improves the safety and durability of environmental structures exposed to highvelocity fluid flow and causes the noise and vibration to be decreased as well.

Extensive research has been devoted in the past years to investigate the drag coefficient of various objects (Jones et al. 1969; Koide et al. 2006; Kumar and Subramanian 
2007; Terry and Barber 2007; Tutar and Holdo 2001). Rahman et al. (2007), investigated the flow characteristics around the circular cylinder with special attention to the mean value of the pressure drag for $\operatorname{Re}=100,1,000$, 3,900 . They explained that using standard $\mathrm{k}-\varepsilon$ model led to more accurate results in comparison with the experimental values for $\operatorname{Re}=1,000,3,900$ for computing drag coefficients. They believed that the realizable $\mathrm{k}-\varepsilon$ model was more useful for vortex-shedding observation. The SST ( $\mathrm{k}-$ $\omega)$ model was suggested for high Reynolds numbers. They also showed that these calculated results for $\operatorname{Re}=100$, 3,900 were closer to the experimental results of Anderson (2005) and Lourenco and Shih (1993). Recently, numerical investigation of the drag and lift coefficient has been performed for inclined circular cylinder of Reynolds numbers in the range of 1-40 (Vakil and Green 2009). Sarkar et al. (1997)and lately Jagadeesh et al. (2008) gave a valuable experimental assessment of the hydrodynamic force of autonomous underwater vehicles hull form as well as the experimental investigation of Sayer (1996) on remotely operated vehicles, added by studying the performance of such structures achieved from numerous cases. Likewise some experiments were implemented in sequence to investigate the effect of cavitation on the drag force of circular cylinder (Jafari Gavzan and Rad 2009). Numerical approaches have also been taken into consideration to evaluate the viscous drag forces of underwater vehicles (Karim et al. 2008) while Lin et al. (1995) computed that for ship hull geometry. Similarly, Sung et al. (1997) made a great progress as a crucial state to the precise prediction of forces and moments of actual axisymmetric bodies by means of computational fluid dynamics (CFD) analysis. Most of the previous works in this field of study were restricted to the flow investigation of the bluff bodies (Aiba 1998; Bearman 1997; Cheng and Liu 2000; Matsuda et al. 2003; Zasso et al. 2006). Kim and Morris (2002) presented the numerical simulation of compressible inviscid mean flow pattern of a cone with $60^{\circ}$ vertex angle. The results were compared to the experimental data, assessed by Calvert (1967). Sakai (2009) considered truncated cones to evaluate the CFD simulation of the drag performance of supersonic flow with repetitive energy depositions. Some experimental investigations have also been implemented in a towing tank to evaluate the hydrodynamic coefficients (Alvarez et al. 2009; Bong-Huan et al. 2009; Jiaming et al. 2005). More recently, (Niknafs 2010) studied the influence of Reynolds number $\left(1.7 \times 10^{5} \leq \operatorname{Re} \leq 6.45 \times 10^{5}\right)$ on drag coefficient of tubular frustum of $10^{\circ}$ vertex angle, by means of performing some experiments in a water towing tank. However, he did not investigate the flow pattern in detail for such Reynolds numbers.

Although tubular-truncated cone have various applications such as in aerospace or underwater structures, turbines, conduits and channels, etc., this type of body has received less attention in the previous research works. Hence, in this research, a typical divergent tubular-truncated cone of $16^{\circ}$ vertex angle is studied experimentally and numerically. A shape optimization study is also performed on the above-mentioned model in order to minimize the drag coefficient. In doing so, a series of wind tunnel experiments was implemented and the drag coefficients were measured during the tests. The numerical procedure, meshing and code validation are also provided in the following section. The flow characteristics consist of the drag coefficient and its components, the velocity and static pressure profiles are analyzed in the numerical simulations as well. For this purpose, the model was subjected to the incompressible airflow where the dimensionless Reynolds number defined as:

$\operatorname{Re}=\rho U_{\infty} L / \mu$; where, $\rho$ fluid density $\left(\mathrm{kg} / \mathrm{m}^{3}\right), U_{\infty}$ free upstream velocity $\left(\mathrm{m} / \mathrm{s}^{2}\right), L$ length of the model $(\mathrm{m}), \mu$ dynamic viscosity $\left(\mathrm{kg} / \mathrm{ms}^{2}\right)$.

The considered non-dimensional drag coefficient $\left(C_{\mathrm{D}}\right)$ and its components: the pressure drag coefficient $\left(C_{\mathrm{DP}}\right)$ and the viscous drag coefficient $\left(C_{\mathrm{DV}}\right)$ are defined as:

$$
\begin{aligned}
& C_{\mathrm{D}}=\frac{F_{\mathrm{D}}}{\frac{1}{2} \rho U_{\infty}^{2} A} \\
& C_{\mathrm{DP}}=\frac{F_{\mathrm{DP}}}{\frac{1}{2} \rho U_{\infty}^{2} A} \quad C_{\mathrm{DV}}=\frac{F_{\mathrm{DV}}}{\frac{1}{2} \rho U_{\infty}^{2} A}, \quad C_{\mathrm{D}}=C_{\mathrm{DP}}+C_{\mathrm{DV}}
\end{aligned}
$$

where $F_{\mathrm{D}}$ is drag force $(\mathrm{N}), F_{\mathrm{DP}}$ pressure drag force $(\mathrm{N})$, $F_{\mathrm{DV}}$ viscous drag force $(\mathrm{N}), \rho$ fluid density $\left(\mathrm{kg} / \mathrm{m}^{3}\right), U_{\infty}$ free upstream velocity $\left(\mathrm{m} / \mathrm{s}^{2}\right)$, and $A$ frontal area $\left(\mathrm{m}^{2}\right)$.

Finally, further investigations on various modified shapes of the divergent tubular-truncated cone along with derived conclusions round out the article. The experimental wind tunnel tests were conducted in the Aerodynamic Laboratory of the IAU, Science and Research Branch in Tehran from 2009 to 2010.

\section{Materials and methods}

Models of the divergent tubular-truncated cone

The models used for the current study are classified into two types: the primary model and the modified models. The primary model used for experimental measurements and computer simulations is a divergent tubular-truncated cone as sketched in Fig. 1. The model is made of plexiglass with a half-vertex angle, $\alpha$, of $8^{\circ}$ and a base diameter $(D)$ of $81.5 \mathrm{~mm}$. Comparative measurements showed that selecting a base diameter of $81.5 \mathrm{~mm}$, about one-fourth of the test section width and height, are adoptable so as to reduce the effect of lateral walls on the flow around the model and 
provide a uniform far field flow. The other dimensions, $d$ and $L$, (Fig. 1) of the model are 34 and $171.4 \mathrm{~mm}$, respectively.

The modified models, made as a function of inclination angle $(\theta)$ are illustrated in Table 1 . The angle, $\theta$, is the angle varies between $0^{\circ}$ and $45^{\circ}$ while the inlet and outlet span diameters remain constant. Abbreviated names were used by means of introducing the symbols of $\mathrm{T}, \mathrm{T} 0, \mathrm{~T} 10, \ldots \ldots$ instead of their full names in order to facilitate referring the models (both primary and modified models) in the entire manuscript, as shown and specified clearly in Table 1.

\section{Experimental procedure and measurements}

It is known that the computer simulations are not favorable unless validated experimentally. In this regard, a series of experimental tests was implemented in a wind tunnel. The experimental results of drag coefficients were obtained under carefully controlled laboratory conditions, in an open circuit wind tunnel with an octahedral test section of $311 \mathrm{~mm}$ (width) $\times 311 \mathrm{~mm}$ (height) and $460 \mathrm{~mm}$ (length) The Re varied from $4.7 \times 10^{4}$ up to $1.94 \times 10^{5}$ based on the model length $(L)$. It was assumed that all the flow patterns around the model were symmetrical about the centerline, irrespective of their axial position. A digital balance system was used for drag forces data collection in considered $\mathrm{Re}$ numbers obtained by changing airflow velocity. All results for each data report were measured under similar and constant laboratory conditions.

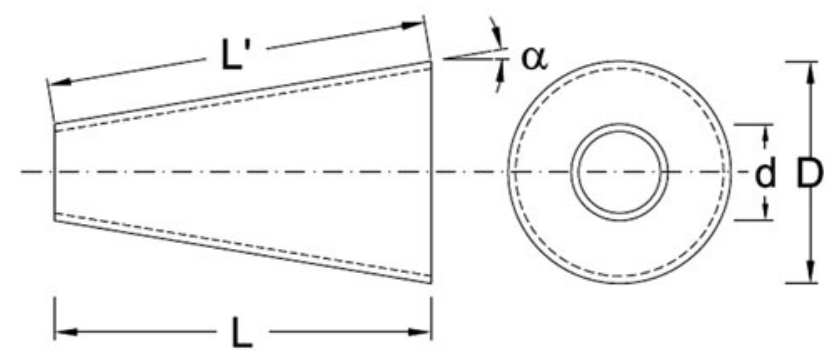

Fig. 1 Schematic view of the primary model
Numerical simulation procedure

The width of the side boundaries of the computational domain used for numerical simulation is $24 \mathrm{~L}^{\prime}$. Both top and bottom slip lateral walls are extended $10 \mathrm{~L}^{\prime}$ from the axis. It should be noticed that these dimensions of the computational domain are carried out in view of obtaining uniform far field velocity. The domain independency test is studied for several domains to ensure the fineness of the computational grid spacing.

The computational domain consists of two different mesh types. Owing to the high velocity gradient in the very thin boundary layer of the model surfaces, mesh fineness near the surfaces are increased. Moreover, the unstructured finer mesh type is used near the both external and internal surfaces of the model whereas the quadrilateral-structured mesh type is used far from the surfaces. A grid independency test is also performed to make sure that the solution is independent of grid size.

The slip wall boundary condition is used for the top and bottom lateral walls. Uniform velocity is considered for inlet boundary while a pressure outlet boundary condition is used at flow outlet. Moreover, the no slip walls are used as model surfaces boundary conditions here.

It is interesting to analyze the flow characteristics over different shape bodies using two-dimensional approach, a well known and mostly used for computer simulation. It reduces computational time extremely and captures the flow pattern as well as three-dimensional analysis. Flow behavior of this specified model is evaluated through finite volume numerical analysis. It is also presumed that the flow behavior is axisymmetric and there is no circumferential flow. The CFD package FLUENT 6.3.26 (2006) is employed using $\mathrm{K}-\varepsilon$ turbulent model with special attention to the steady state, viscous, incompressible flow for the range of Reynolds numbers between $9.41 \times 10^{2}$ and $1.882 \times 10^{7}$. The SIMPLE algorithm is used to link the pressure and velocity adequately. Second-order upwind discretization gives better accuracy, while first-order discretization produces more

Table 1 Different models used for numerical simulations

\begin{tabular}{|c|c|c|c|c|c|c|c|}
\hline Specification & Primary model & $\theta=0^{\circ}$ & $\theta=10^{\circ}$ & $\theta=20^{\circ}$ & $\theta=30^{\circ}$ & $\theta=40^{\circ}$ & $\theta=45^{\circ}$ \\
\hline Model Name & $T$ & T0 & T10 & T20 & T30 & T40 & T45 \\
\hline Configuration & & & & & & & \\
\hline
\end{tabular}

Note: $\theta$ is the inclination angle used for modified models design:

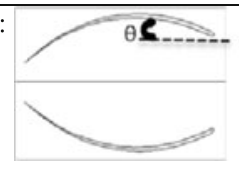




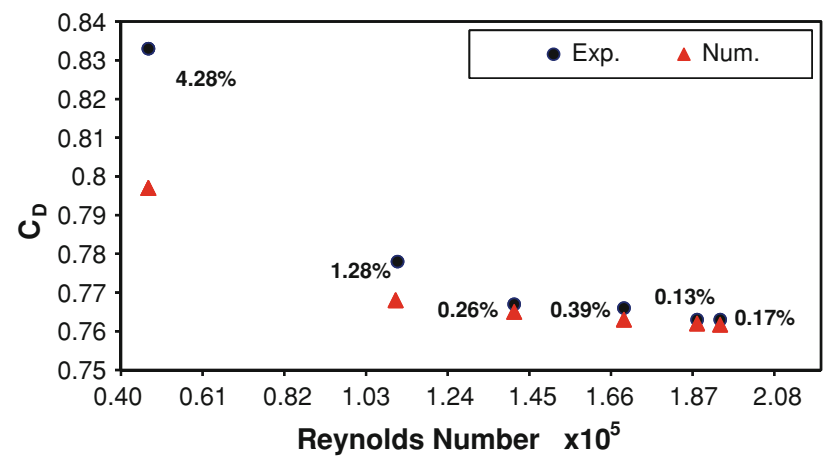

Fig. 2 Drag coefficient versus Re for the primary model

robust convergence. In the current study, when the secondorder scheme did not converge, the first-order scheme was used for starting the iterations and then the iterations were continued by switching to the second-order scheme. In addition, the convergence criteria in the order of $10^{-6}$ are adopted in this study for all the solution residues.

\section{Code validation}

To verify this work, the results of an experimental study of drag coefficient around the primary truncated cone of

(a)

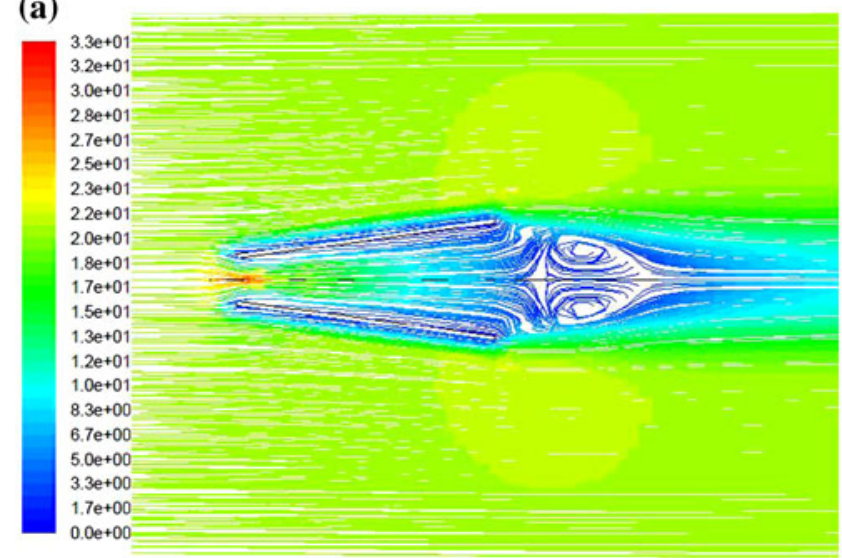

(c)

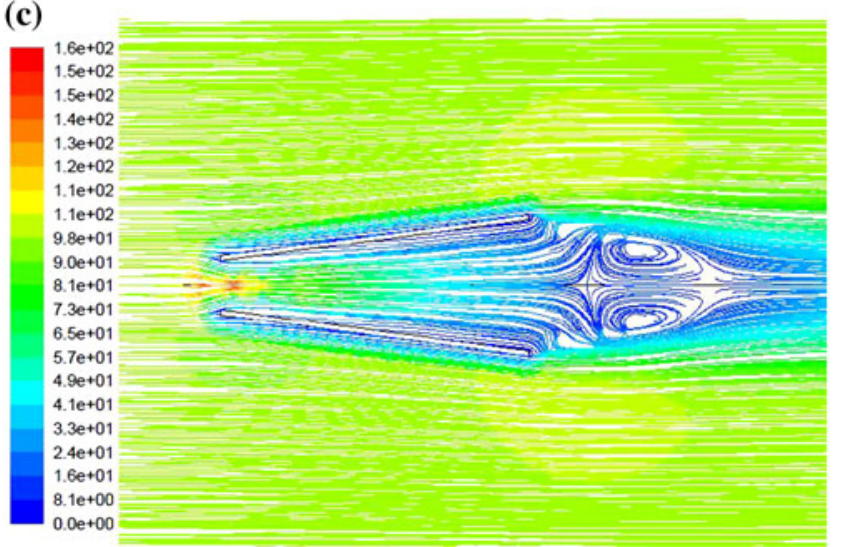

$16^{\circ}$ vertex angle within the range of $\mathrm{Re}$ between $4.7 \times 10^{4}$ and $1.94 \times 10^{5}$, are confronted with its corresponding numerical-simulated values, as shown in Fig. 2. The percentage error between computational and experimental results for each Re is also depicted in Fig. 2. The comparison shows that the average error is equal to $1.09 \%$. This Figure also illustrates that the experimental and numerical results compare well which confirms code validation.

\section{Results and discussion}

Flow around and through the primary model

In the case of truncated cone of $16^{\circ}$ vertex angle (model T), the slowing of the velocity which causes an unfavorable pressure gradient is sufficient to separate the flow behind the model. These two axisymmetric formed bubbles are shown clearly in Fig. 3a, for $\operatorname{Re}=1.94 \times 10^{5}$. As Re increases, the flow progresses and two bubbles become more aggressive and forming two enclosed recirculating bubbles, shown in Fig. $3 \mathrm{c}$ for turbulent flow of $\operatorname{Re}=1.882 \times 10^{7}$, as well. The complete outline of

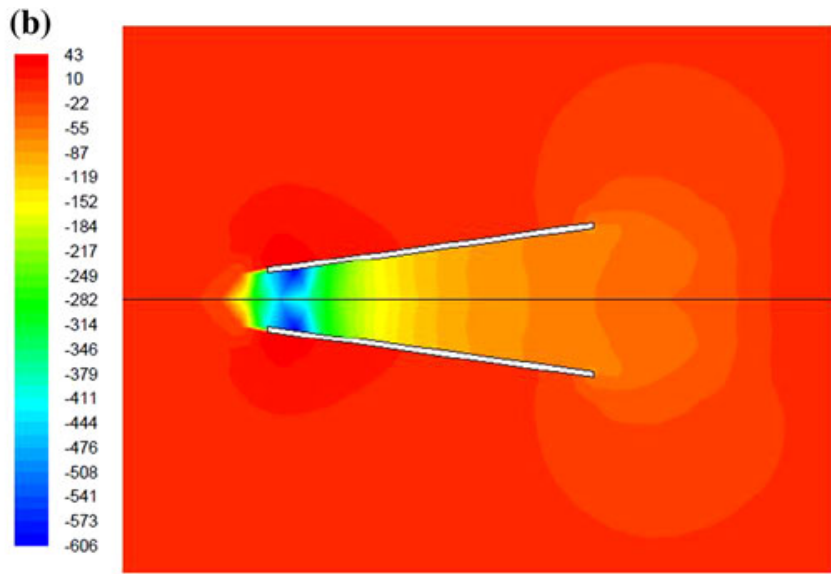

(d)

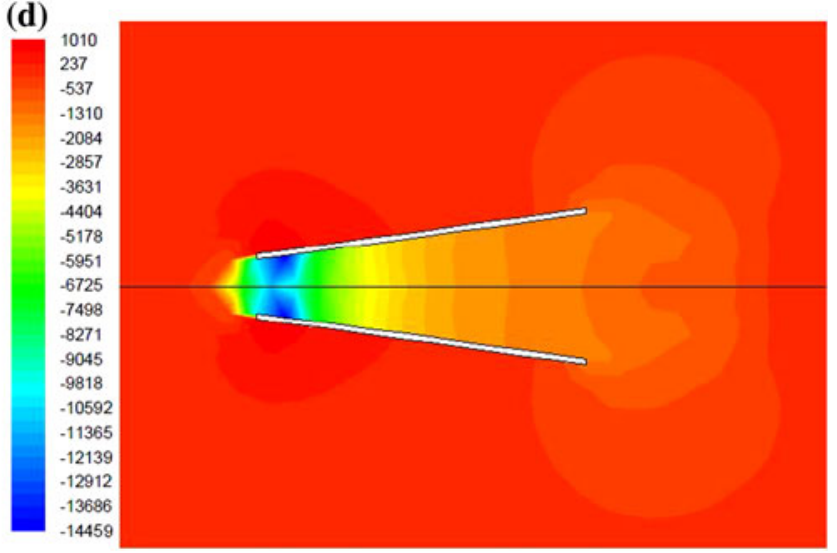

Fig. 3 a, c Pathline; b, d static pressure of model T. a, b Re $=1.94 \times 10^{5} ; \mathbf{c}, \mathbf{d} \operatorname{Re}=1.882 \times 10^{7}$ 
(a)

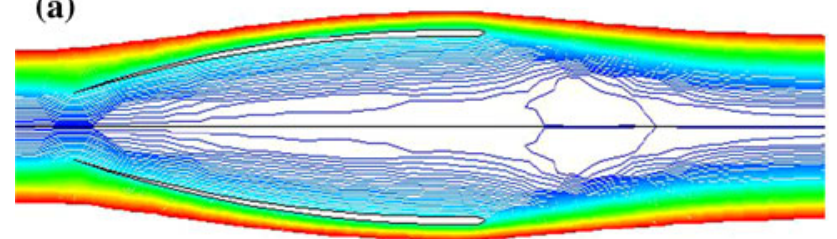

(b)

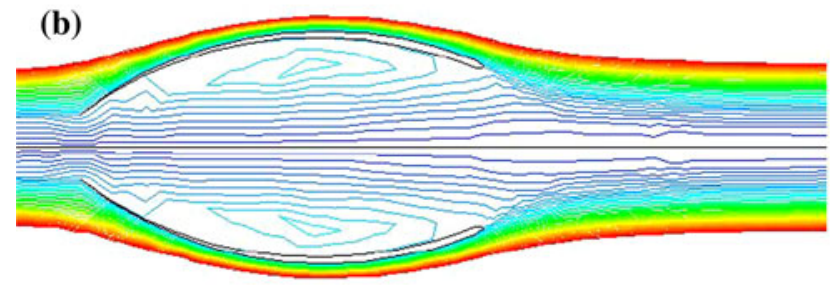

(c)
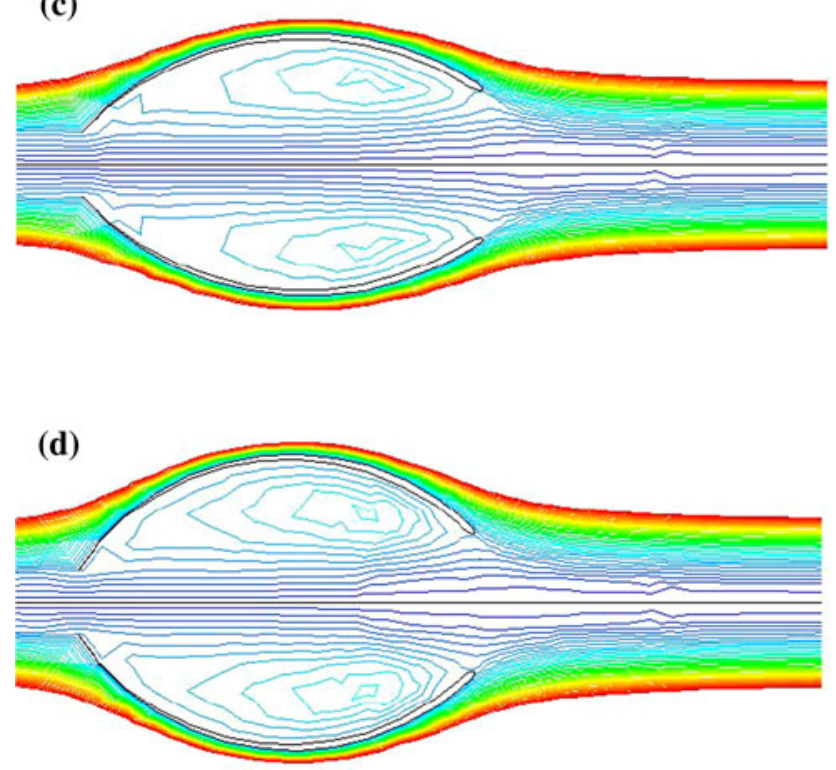

(e)

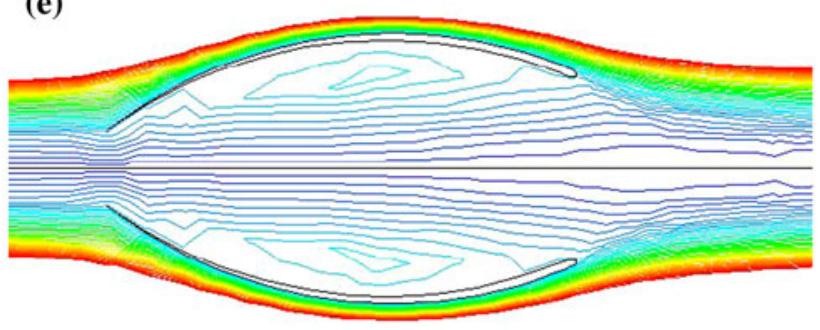

(f)

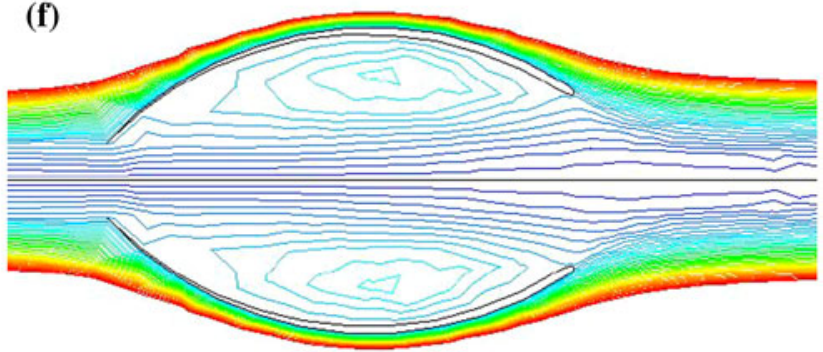

(g)

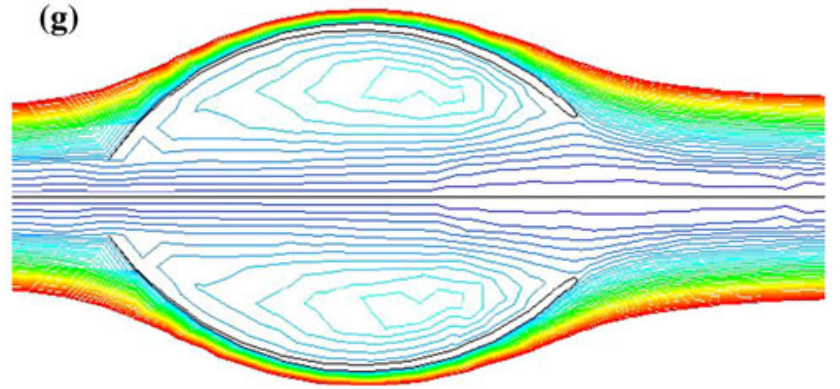

(h)

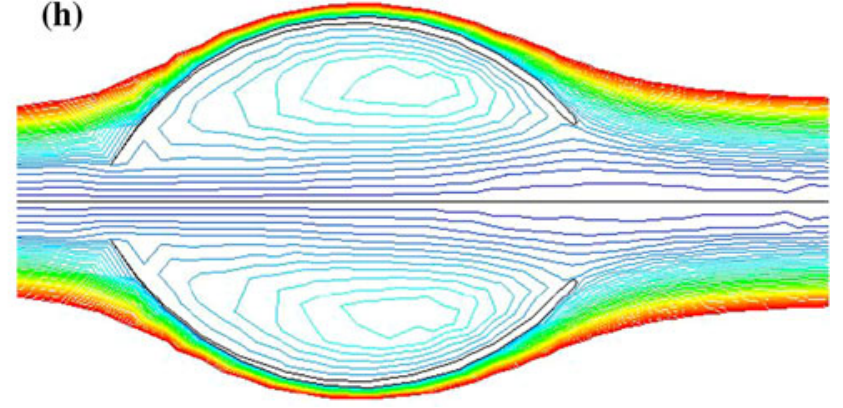

Fig. 4 Streamlines around and through the model a T0, b T20, c T30, d T40 while Re $=1.882 \times 10^{7}$; and for models e T20, f T30, g T40, h T45 while $\operatorname{Re}=1.94 \times 10^{5}$

correlated static pressure distribution is presented in Fig. 3b, d for both laminar and turbulent flows, respectively.

Flow around and through the modified models

On the other hand, for the modified shapes where the flow separation occurs inside the model, the flow behavior is much more complex than its related primary shape. In this regard, it is noteworthy to explain the desired flow characteristics by means of several streamlines that are investigated for different types of flows. To some extent, similar flow patterns are observed either for laminar or turbulent flow regime. To expound better the ambiguity of the drag coefficient performance, the streamlines of the flow are plotted for representative modified models in Fig. 4, for typical Reynolds numbers of $1.882 \times 10^{7}$ and $1.94 \times 10^{5}$, respectively.

Figure 4a represents the flow pattern of model T0. It is observed that in spite of streamlining, two axisymmetric bubbles still exist at decreasing distance from the outlet span behind the model. They are decreasing in size in comparison with size of the bubbles formed behind the model $\mathrm{T}$. Thereafter, as $\theta$ increases to $20^{\circ}$, the flow passes 


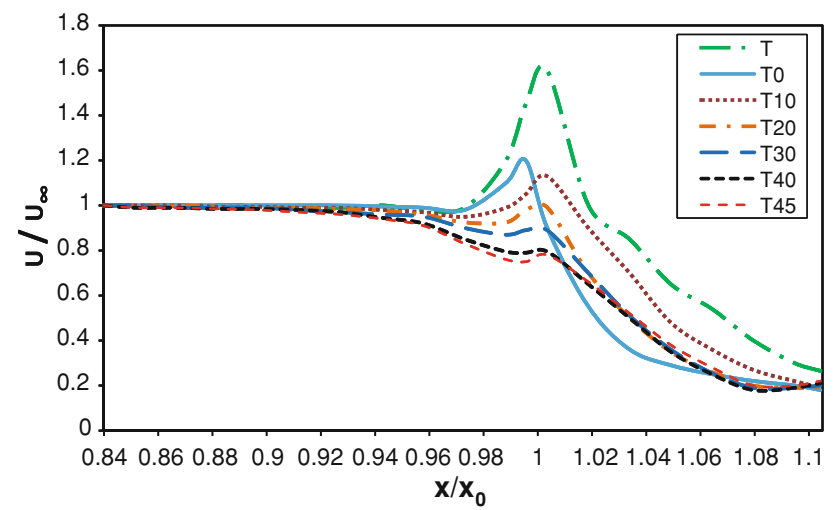

Fig. 5 Non-dimensional velocity profile along the axis of both primary and modified models

slightly through the model T20. Under such circumstances, the wake itself disappears completely behind the model while the separation occurs near the internal surface, presented in Fig. 4b, e. (the minimum drag forces obtained at the same $\theta$ ).

The evolution of aforementioned bubbles as a function of internal area expansion is certified in Fig. 4c, d, f, g, and $\mathrm{h}$. Although the width of the recirculating zone is about the model length $(L)$, the centers of the two standing eddies begin to shift a little toward downstream, as seen in Fig. $4 c, d, g$, and d. This happens as a result of inside crosssectional area expansion, which causes pressure to be increased headed for downstream.

\section{Velocity profile}

Figure 5 represents such streamwise velocity profiles achieved from the upstream of the fluid flow to the outlet span along the model axis. The $X$ coordinate of the point on the center of the inlet span is defined as $X_{0}$. The velocity profile of model $\mathrm{T}$ is plotted along with those of the modified models. It is deduced from the graph that the maximum velocity inside the inlet span close to the entrance increases by about $60 \%$ compared with the free upstream velocity magnitude in model $\mathrm{T}$. Thereafter, it decreases abruptly from the peak toward the outlet span. The considerable increase in velocity could be used as an important source of energy for various applications. It is also indicated that by increasing $\theta$ from $0^{\circ}$ to $45^{\circ}$, the peak point in the velocity profile is diminishing owing to the inside sectional area expansion.

\section{Drag coefficients}

In the following, the influence of Re on drag coefficients of both primary and modified tubular-truncated cones is evaluated by the numerical simulation.

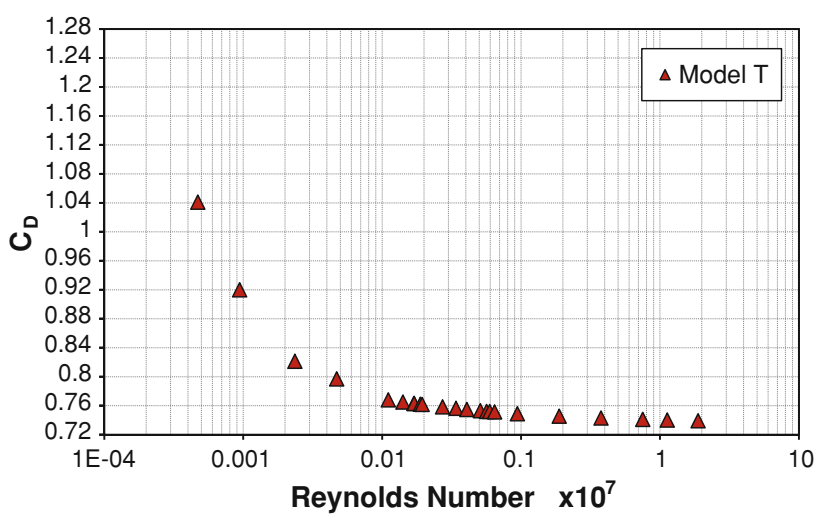

Fig. 6 Computed drag coefficient versus Re for model $\mathrm{T}$

On the occasion that a divergent tubular-truncated cone is oriented to the flow, a frontal area becomes hollow substituted successively in simulations for $C_{\mathrm{D}}$ calculations. The reduction of $C_{\mathrm{D}}$ against increasing $\mathrm{Re}$ for $9.41 \times 10^{2} \leq \operatorname{Re} \leq 1.882 \times 10^{7}$ is obvious in Fig. 6. The figure reveals that the $C_{\mathrm{D}}$ value of model T decreased by $42 \%$ as a function of Re in the range of $9.41 \times 10^{2}$ up to $1.882 \times 10^{7}$ with reference to the $\operatorname{Re}=9.41 \times 10^{2}$.

For better explanation the issue, the static pressure distribution graphs inside the model $\mathrm{T}$ along the axis of the model are presented in Fig. 7 for various Re. The pressure drag is usually dominant for this type of body (Cengel and Cimbala 2010). Therefore, the main reason for the drag increase with Re which is due to the pressure difference between the front and back sides of the model is clearly shown in this figure as a function of Re.

Table 2 provides detailed investigation intended for the contribution of pressure and viscous drag coefficients, $C_{\mathrm{DP}}$ and $C_{\mathrm{DV}}$, to the total drag coefficient, $C_{\mathrm{D}}$, for model $\mathrm{T}$ along with the modified models, for two representative Re of $1.94 \times 10^{5}$ and $1.882 \times 10^{7}$. Moreover, the pressure coefficient, $C_{\mathrm{DP}}$, dominance and its decrease comparable to

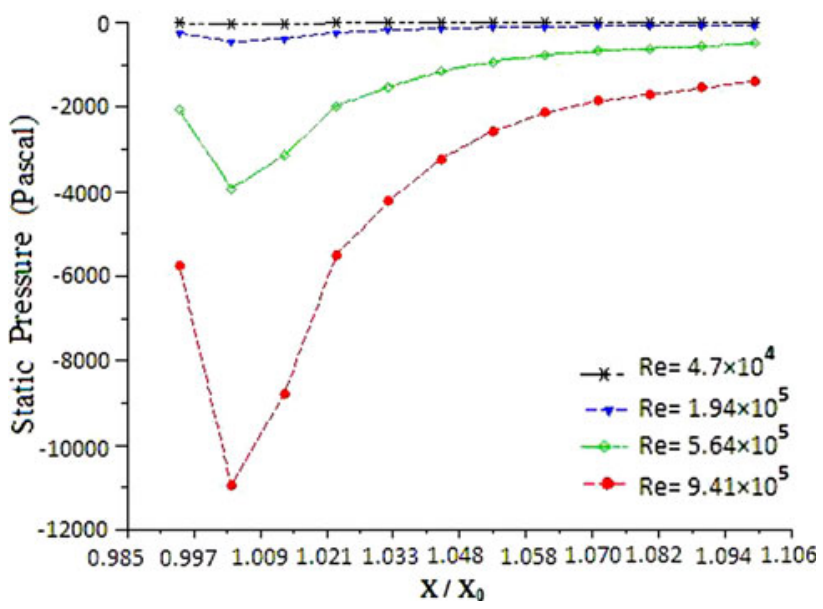

Fig. 7 Static pressure distribution along the axis of model $\mathrm{T}$ 
Table 2 Contribution of the pressure and viscous drag coefficients to total drag coefficient calculated for various models

\begin{tabular}{lllllllll}
\hline $\operatorname{Re}$ & $C_{\mathrm{DP}}$ and $C_{\mathrm{DV}}$ & $\mathrm{T}$ & $\mathrm{T} 0$ & $\mathrm{~T} 10$ & $\mathrm{~T} 20$ & $\mathrm{~T} 30$ & $\mathrm{~T} 40$ & $\mathrm{~T} 45$ \\
\hline $1.94 \times 10^{5}$ & $C_{\mathrm{DP}}$ & 0.7290 & 0.4253 & 0.3235 & 0.2422 & 0.2305 & 0.2306 & 0.2442 \\
& $C_{\mathrm{DV}}$ & 0.0326 & 0.0363 & 0.0282 & 0.0231 & 0.01969 & 0.0164 & 0.0153 \\
$1.882 \times 10^{7}$ & $C_{\mathrm{DP}}$ & 0.7305 & 0.4174 & 0.3099 & 0.2324 & 0.2179 & 0.2229 & 0.2379 \\
& $C_{\mathrm{DV}}$ & 0.0087 & 0.0087 & 0.0067 & 0.0058 & 0.005 & 0.0045 & 0.0045 \\
\hline
\end{tabular}

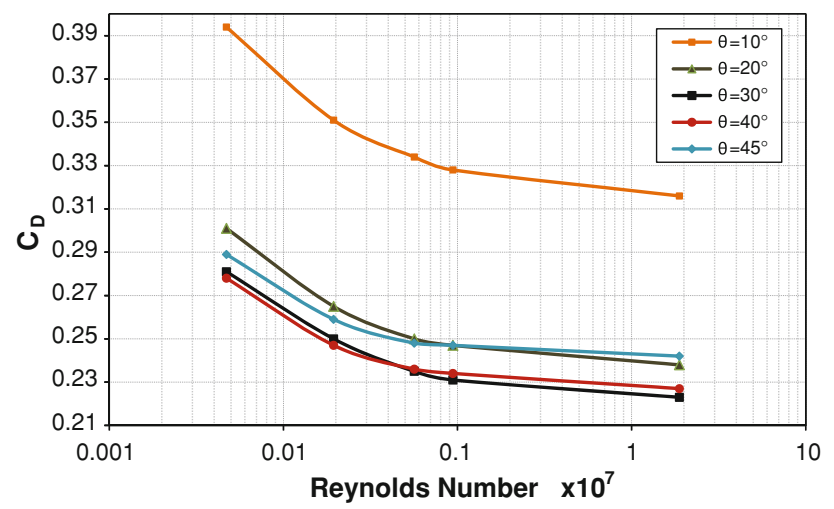

Fig. 8 Influence of Re on drag coefficient for various inclination angles

Table 3 Comparison of drag coefficient between models T, T0 and the optimum model

\begin{tabular}{llll}
\hline $\operatorname{Re}$ & Model T & Model T0 & Optimum model \\
\hline $4.7 \times 10^{4}$ & 0.797 & 0.512 & 0.278 \\
$1.94 \times 10^{5}$ & 0.761 & 0.461 & 0.247 \\
$5.64 \times 10^{5}$ & 0.752 & 0.444 & 0.235 \\
$9.41 \times 10^{5}$ & 0.748 & 0.44 & 0.231 \\
$1.882 \times 10^{7}$ & 0.739 & 0.426 & 0.223 \\
\hline
\end{tabular}

$C_{\mathrm{DV}}$ considerable diminution with Re which leads to the $C_{\mathrm{D}}$ reduction as a function of $\mathrm{Re}$, are included in this table for better understanding the concepts discussed previously.

Figure 8 presents an exponential decrease of drag coefficient versus Re between $4.7 \times 10^{4}$ and $1.882 \times 10^{7}$, for different angles of inclination. It is deduced from the figure that the minimum $C_{\mathrm{D}}$ is obtained at $\theta=40^{\circ}$ for Re less than about $5.2 \times 10^{5}$ while thereafter, $\theta=30^{\circ}$ is achieved as an optimum $\theta$ to minimize the drag coefficient.

The values of Table 3 shows that $C_{\mathrm{D}}$ reduces for both optimum and T0 models in comparison with that of model $\mathrm{T}$ in the range of Re from $4.7 \times 10^{4}$ up to $1.882 \times 10^{7}$. Moreover, the reduction of $C_{\mathrm{D}}$ comparable to that of model $\mathrm{T}$ increases with Re. The maximum increase in $C_{\mathrm{D}}$ is 42.3 and $69.8 \%$ at the highest Re of $1.882 \times 10^{7}$ for model T0 and the optimum model, respectively. This reduction is attributed to the fact that as $\theta$ increases in order to configure an optimum shape, both correlated pressure and viscous coefficients decrease noticeably and let the total $C_{\mathrm{D}}$ to be decreased, as well.

\section{Conclusion}

There is a growing interest in reduction of drag coefficient over various bodies and its considerable effect on fuel and energy consumption as a main scope of efficiency improvement in energy management. It also causes a noticeable decrease in environmental pollution. Accordingly, a CFD study of flow over and through the primary tubular divergent-truncated cone and the proposed modified shapes has been particularly performed to identify possible optimum shape of minimum drag coefficient for energy efficiency case study. In doing so, the comparison of numerical results with experimental data, which were conducted in a wind tunnel, represented the capability and accuracy of the computer simulations in an idealized case study. The influence of pressure and viscous drag coefficients to total drag coefficient were also interpreted for better understanding. Computational simulations revealed that $C_{\mathrm{D}}$ reduces more effectively for the optimum model at approximately $\theta=30^{\circ}$ for the range of $\operatorname{Re}$ of laminar flow. For turbulent flow, typically at $\mathrm{Re}$ in the range of $5.2 \times 10^{5}$ up to $1.882 \times 10^{7}$ the optimum model procured at $\theta=40^{\circ}$. Consequently, the maximum drag reduction of about $69.8 \%$ at the highest considered Re of $1.882 \times 10^{7}$ was obtained through the numerical simulations which is a significant consequence for the applications in which $C_{\mathrm{D}}$ is important. In addition, the streamwise velocity profiles for different cases of primary and modified models were studied. The research led to the fact that the maximum velocity inside the inlet span close to the entrance increases by $60 \%$ compared to the free upstream velocity magnitude in the primary model. This can be used to supply either some devices or apartments lightening-system power requirement by means of using generators or some other generating energy equipments. These results illustrate a unique case for the use of in applications related to the energy management case study. 
Acknowledgments The authors would like to express their gratitude toward the authorities of IAU, Science and Research Aerodynamic Laboratory, where the experimental tests were performed.

\section{References}

Aiba S (1998) Flow dynamic drag of an axially symmetrical bluff body consisting of a plane surface and a spherical surface. J. Fluids Eng 120(4):851-853

Alvarez A, Bertram V, Gualdesi L (2009) Hull hydrodynamic optimization of autonomous underwater vehicles operating at snorkeling depth. J Ocean Eng 36(1):105-112

Anderson JD (2005) Fundamentals of Aerodynamics, 4th edn. McGrow Hill Inc, Columbus

Bearman PW (1997) Near wake flows behind two- and threedimensional bluff bodies. J Wind Eng Ind Aerodyn 69-71(1):33-54

Bong-Huan J, Jin-Yeong P, Fill-Youb L, Pan-Mook L, Chong-Moo L, Kihun K, Young-Kon L, Jun-Ho O (2009) Development of the AUV 'ISMI' and a free running test in an ocean engineering basin. J Ocean Eng 36(1):2-14

Calvert JR (1967) Experiments on the low speed flow past cones. J Fluid Mech 27(2):273-289

Cengel YA, Cimbala JM (2010) Fluid mechanics: fundamentals and applications, 2nd edn. McGrow Hill Inc., New york, pp 580-590

Cheng M, Liu GR (2000) Effect of after body shape on flow around prismatic cylinders. J Wind Eng Ind Aerodyn 84(2):181-196

Fluent 6.3 User's Guide (2006) Fluent Inc., Lebanon, p 03766

Jafari Gavzan I, Rad M (2009) Experimental analysis of cavitation effects on drag force and back pressure of circular cylinder with free turbulence. Sci Iran 16(4):344-353

Jagadeesh P, Murali K, Idichandy VG (2008) Experimental investigation of hydrodynamic force coefficients over AUV hull form. J Ocean Eng 36(1):113-118

Jiaming W, Jiawei Y, Cheng Y, Yuanming C, Huiping T, Xiaohui X (2005) Experimental study on a controllable underwater towed system. J Ocean Eng 32(14-15):1803-1817

Jones GW, Cincotta JJ, RWb Walker (1969) Aerodynamic forces on a stationary and oscillating circular cylinder at high Reynolds numbers. NASA Tech Rep R 300:1-62

Karim M, Rahman M, Alim A (2008) Numerical computation of viscous drag for axisymmetric under water vehicles. J. Mek 26(1):9-21

Kim JW, Morris JPh (2002) Computation of subsonic inviscid flow past a cone using high-order scheme. J AIAA 40(10):1961-1968
Koide M, Okanaga H, Aoki K (2006) Drag reduction effect of square cylinders by grooves and corner-cuttings. J Visual Soc Jpn 26(1):69-72

Kumar M, Subramanian A (2007) A numerical and experimental study on tank wall influences in drag estimation. J Ocean Eng 34(1):192-205

Lin CW, Percival S, Gotimer EH (1995) Viscus drag calculations for ship hull geometry. Tech Rep, Design evaluation branch, Hydromechanics directorate, David Taylor Model Basin, Carderock Division Naval Surface Warfare Center, Bethesda, MD, USA

Lourenco LM, Shih C (1993) Characteristics of the plane turbulent near wake of a circular cylinder: a particle image velocimetry study, Private Communication

Matsuda k, Uejima H, Sugimoto T (2003) Wind tunnel test on in-line oscillation of a two-dimensional circular cylinder. J Wind Eng Ind Aerodyn 1-2:83-90

Niknafs AY (2010) Experimental investigation of drag on tubular frustum immersed in a water moving in different directions and variation of drag coefficient. M.Sc. Dissertation, IAU (South Tehran Branch), Iran

Rahman M, Karim M, Alim M (2007) Numerical investigation of unsteady flow past a circular cylinder using 2-D finite volume method. J Navig Archit Mar Eng 4(1):27-42

Sakai T (2009) Supersonic drag performance of truncated cones with repetitive energy depositions. Int J Aerosp Innov 1(1):31-43

Sarkar T, Sayer PG, Fraser SM (1997) A study of autonomous underwater vehicle hull forms using computational fluid dynamics. Int J Numer Meth Fluids 25(11):1301-1313

Sayer P (1996) Hydrodynamics forces on ROV near the air/sea interface. Int J Offshore Polar Eng 6(3):177-183

Sung CH, Fu TC, Griffin MJ, Huang TT (1997) Validation of incompressible flow computation of forces and moments on axisymmetric bodies undergoing constant radius turning. In: Proceedings of the 21 symposium on naval hydrodynamics, pp 1048-1060

Terry J, Barber T (2007) CFD and experimental study of an inflatable re-entry vehicle model at Mach 3 conditions. J Acta Astronautica 61(10):854-865

Tutar M, Holdo AE (2001) Computational modeling of flow around a circular cylinder in sub-critical flow regime with various turbulence models. Int J Numer Meth Fluids 35(1):763-784

Vakil A, Green SI (2009) Drag and lift coefficients of inclined circular cylinders at moderate Reynolds numbers. J Comput Fluids 38(9): 1771-1781

Zasso A, Giappino S, Muggiasca S (2006) Wind tunnel study of a cone-like shaped roof: Reynolds number effects. J Wind Eng Ind Aerodyn 94(5):431-444 